\title{
A Survey on the Status of Smart Healthcare from the Universal Village Perspective
}

\author{
Guoxin Huang \\ Department of Computer Science \\ The University of Arizona \\ Tucson, US \\ gxhuang039@email.arizona.edu
}

\author{
Yajun Fang \\ Computer Science and Artificial \\ Intelligence Laboratory \\ Massachusetts Institute of \\ Technology \\ Cambridge, US \\ yajufang@csail.mit.edu \\ Berthold Horn \\ Computer Science and Artificial \\ Intelligence Laboratory \\ Massachusetts Institute of \\ Technology \\ Cambridge, US \\ bkph@csail.mit.edu
}

\author{
Xiushi Wang \\ Department of Math and \\ Computer Science \\ Lewis \& Clark College \\ Portland, US \\ xiushi.wang@1clark.edu
}

\author{
Yu Pei \\ Department of Arts and Law \\ Beijing University of Chemical \\ Technology \\ Beijing, China \\ peiyu@mail.buct.edu.cn
}

\begin{abstract}
This survey paper discusses the condition of smart healthcare implementation. It discusses the current healthcare problems and how smart healthcare technologies ease the problems. Our group, Universal Village, realizes that the integration and interaction between parties in a system will maximize the effectiveness and benefit for the system. Based on this idea, this paper considers the smart city system as a whole, and talks about how smart healthcare interacts with infrastructures and functions inside and outside of the smart healthcare field. Then, it analyzes how a more powerful integrated system can be built from the smart healthcare system. In the end, several case studies are listed. Based on our analysis and the case studies, this paper then ended with the future prospects of the smart healthcare.
\end{abstract}

Keywords-Universal Village, Smart City, Smart Healthcare, System Dynamic

\section{INTRODUCTION}

With the development of the Internet of Things (IoT), Artificial Intelligence (AI), machine learning, and big data technology, applications of novel electrical technologies are wildly used in the healthcare system. However, even if there are new technologies come up quickly, implementing the technologies, establishing interactions between the technologies, and connections between the human being and technologies are totally different stories. In the body part, this paper will talk about the current states of the healthcare system, the challenges we are facing, how the smart healthcare is involved, and the implementation of the smart healthcare system around the world. As most articles focus on new technologies, we believe that how smart healthcare interact with other functions, infrastructures within and out of the smart healthcare system is not given enough attention.

After introducing the smart healthcare and systematical analysis, a case study will be introduced. Then, the paper will end with a vision on the future prospective of smart healthcare.

\section{HeAlthCARE CURRENT Status AND ChallengeS}

There are 4 primary challenges for the current healthcare system: increasing cost, inconsistent quality, inefficient diagnosing process, and population ages.

\section{A. Increasing Cost}

In most of the countries around the world, the healthcare expenditure increases each year. However, since the GDP of most countries are also increasing, discussing purely the growth of healthcare expenditure doesn't reveal the nature. Here, we apply the index "Current Healthcare Expenditure (CHE) as the percentage of Gross Domestic Product (GDP)" to show the pressure that healthcare put on people.

According to the World Health Organization (WHO), in most of the countries, the Current Healthcare Expenditure (CHE) as the percentage of Gross Domestic Product (GDP) is increasing in recent years [1]. Though people around the world is getting wealthier, the pressure from the healthcare expenditure is even getting larger.

Fig. 1 shows that the CHE as the percentage of GDP for five selected countries is increasing. Full data and data related to other countries can be accessed through [1]. Here, only several representatives developing and developed countries are selected to show the trend.

Decreasing the healthcare expenditure will not only decrease the economic pressure for most of the people, but also potentially save the lives of those who are unable to make full payment of their healthcare spending. 


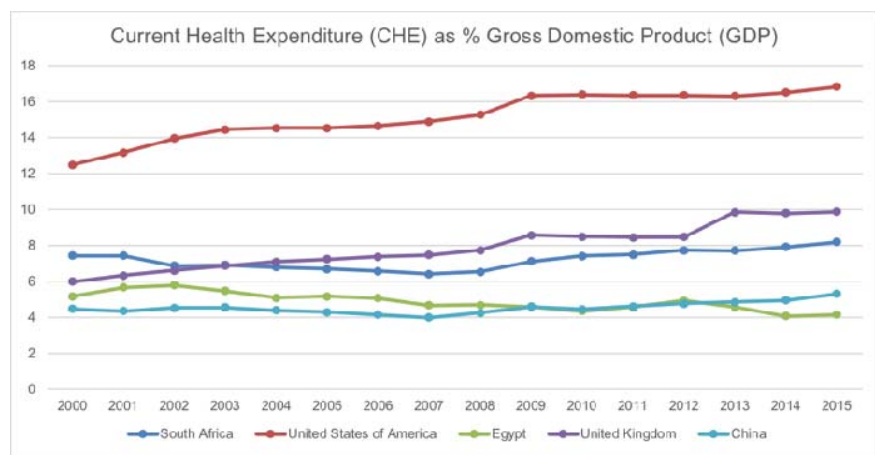

Fig. 1. the CHE as the percentage of GDP for several countries

\section{B. Inconsistent Quality}

Each year, over 30,000 people die due to the deficiency of high-quality healthcare [2]. According to [3], the quality of healthcare consists of six aspects: safety, effectiveness, efficiency, personalizing, timeliness and equitability.

The main cause of the inconsistent quality is lacking good hospitals and professional physicians. Though professional physicians are willing to give more diagnosis and treatment to patients, they are limited by their working time and energy. Hence, in most of the countries, only specific amounts of patients can get the expected diagnosis and treatment.

Lacking high-quality hospitals will result in shortages of efficient healthcare equipment and reliable treating environment. Thus, the safety, effectiveness, efficiency, and timeliness quality are damaged. Nevertheless, diagnosis and treatment from unprofessional physicians could also result in a safety problem, effectiveness problem, efficiency problem, and timeliness problem. The shortage of physicians also damages the personalization and equitability of healthcare. Given that each physician needs to take care of so many patients, especially in developing countries [4], physicians usually need to make a balance between spending time to personalize treatment and taking care of more patients.

In a word, the inconsistent quality is a result of lacking good treating resources and professional physicians.

\section{Inefficient Diagnosing Process}

One of the reasons that cause inefficient diagnosis and treatment process is patients' information retrieving. The diagnosing process always starts with physicians asking required information of patients, especially after the patients are introduced to a new hospital or a new physician. The required information includes both treating-related and nontreating-related information. Since the information usually maintains the same within a certain time, the time spent to repeatedly asking and recording patients' information could be saved if there is a secure information sharing platform.

Another reason causing inefficient diagnosing process is lacking the use of previous diagnosing information. A lot of times, patients are required to do new tests because of information lost. The loss could be a result of lacking of communication when a patient is introduced to a new hospital, or a new physician, or human error, management, and system error. These process waste both the time of the patient and the time of the physicians. As a result, it causes inefficient diagnosing process.

\section{Population Ages.}

The world population ages [5] will bring changes to the current healthcare system in its focus on disease type and caring method. The aged people are more likely to contract diseases such as arteriosclerosis, cardiovascular diseases, heart disease, cancers, osteoporosis, stroke, chronic renal failure, and diabetes. Also, the aged population needs more caring physically and psychologically since they are more likely to live alone, while the diseases are truly threatening them. In this case, both automatic monitoring and psychological caring are required to enhance the aged population's life quality.

Due to the increase of certain disease types caused by population aging, there will be further physician deficiency, especially professional physicians for the diseases mentioned above. Nevertheless, the traditional healthcare system doesn't take too much consideration of multitudinous remote health condition monitoring, diseases condition monitoring, and psychological caring.

\section{SMART HealthCARE SYSTEM}

Part III and part IV can be illustrated in figure 2.

\section{A. Introduction of Smart Healthcare}

Smart healthcare is a term referring to an integrated system where patients, monitors, physicians, Artificial Intelligence, and other parts are connected and interact with each other to personalize healthcare, increase healthcare quality, and decrease cost. Though there is not a smart healthcare designed to purely assist physicians or purely assist patients, based on the function, smart healthcare systems could be divided into 2 types: physician-assisting smart healthcare system and patientassisting smart healthcare. Here, each type will be discussed separately.

\section{B. Smart Healthcare History}

From the end of the 20th century, there appears censorbased monitoring method [6], and device-based patient management platform [7], which could seem as the prototype of the smart healthcare device and system. In the 21 st century, as the computing power of devices increases exponentially, big data analysis, sensors, computing technology, and Internet of Thing (IoT) develop fast. Smart healthcare, then, was leveraged by these technologies, and become more popular and powerful.

\section{Physician-assisting Smart Healthcare System}




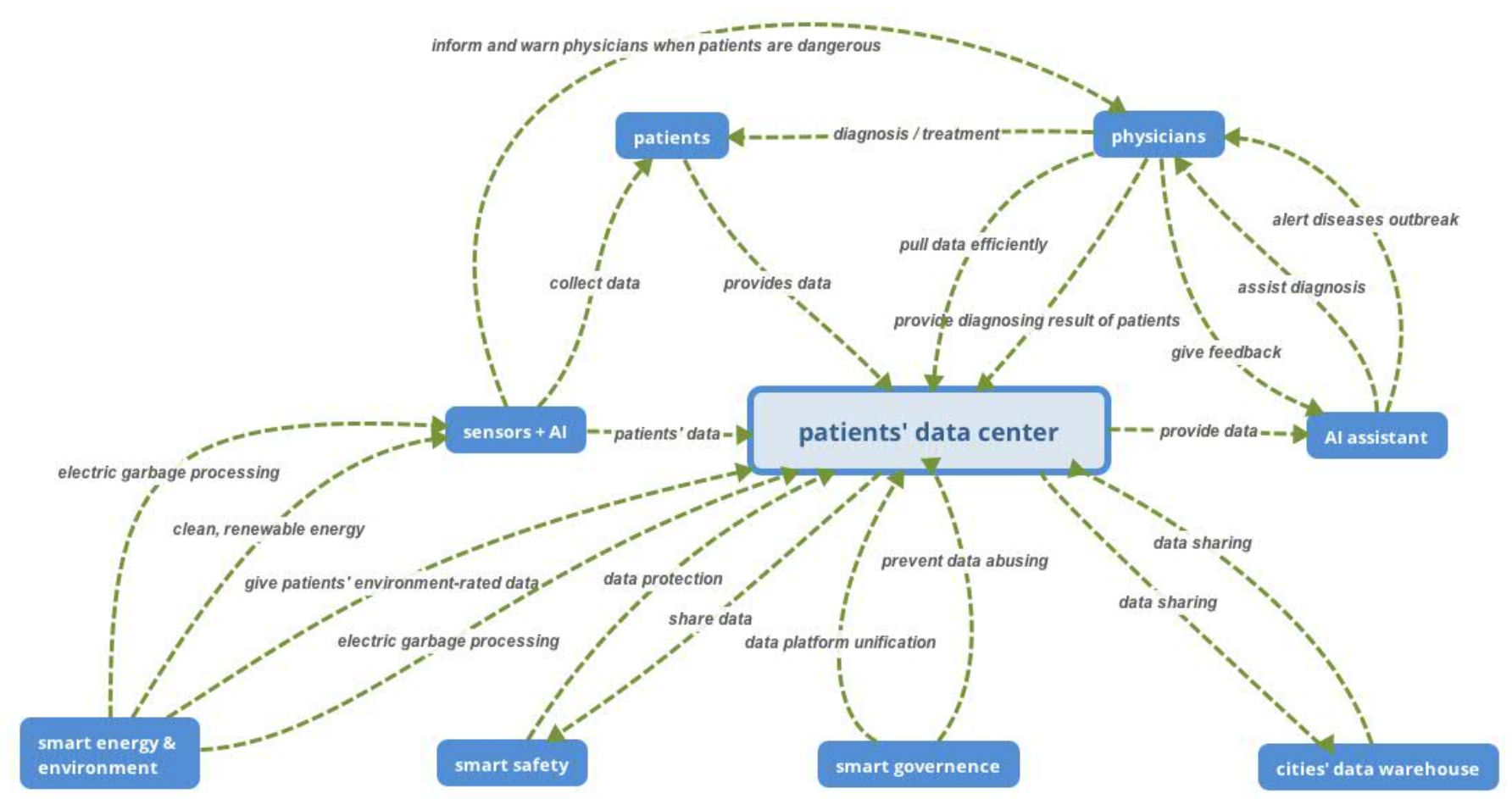

Fig. 2. Interaction between smart healthcare entities and other infrastructures

Physician-assisting smart healthcare system aims at increasing the effectiveness, efficiency, and decrease error rate of diagnosing and treating process. There are lots of platforms discussed in different papers [8-12]. But the basic frameworks of this type of smart healthcare system are similar. Here, a detailed platform will be introduced and analyzed.

\section{Patients Data Sharing}

To start with, a good patient information sharing system is crucial for the smart healthcare system. The platform should be cross hospital, and even cross countries if possible. It should also be safe, fast, and efficient to use.

The system benefits both patients and physicians. With this system, physicians can store and pull the information of patients with their permissions. In this case, the time spent to repeatedly ask patients' information will be saved. Not only patients will spend less time in their diagnosing process, but also the physicians can save their time to save more people. Nevertheless, the reduction of time-consuming will lead to a reduction in money-consuming.

\section{AI-based Diagnosing and Treating Assistant}

With the collected patients' data and diagnosing results put by physicians, big data, computer vision, artificial intelligence (AI), and machine learning techniques can be applied to create AI-based diagnosing and treatment assistant, and then leverage future diagnosing accuracy.

As mentioned in the "inconsistent quality" part above, the diagnosing and treatment given to patients has a big chance to have low quality due to the limitation of the number of professional physicians, and the fact that even the best physicians could have human error when they are tired after long time working.

The AI assistant gives suggestions as references when physicians diagnosis and give treatment to patients. Such assistant saves physicians energy and time. Furthermore, it could save lives when physicians give wrong diagnosing. Physicians will under less pressure with an effective AI assistant; Accidence rate will definitely be decreased with the assistance of AI assistant. As a result, the healthcare will be more efficient, more effective, and less costly.

\section{Disease Prevention Using Big Data Analysis}

As the patients' data are collected into the data pool, machine learning, big data techniques, and visualization techniques can be used to see, predict, and even prevent diseases from outbreaking.

Appropriate analysis can also find connections between certain diseases and certain areas, ages, living habits, environmental condition, and other features. In this case, certain diseases can be prevented.

\section{Remote Healthcare}

In the rural area and other areas where professional physicians are lacked, remote diagnosis and treatment are important. Traveling for healthcare not only costs people's time and money, it could also make people tired. One specific example is the elder. Frequent traveling could be destructive for the elder with diseases. Remote diagnosis and treating are crucial for them. 
The difference between diagnosing face to face and remotely is mainly an information difference. With enough information collected, it is possible and feasible to do remote diagnosis and remotely treatment. The way to ensure insurance information is to use the information sharing platform mentioned above, as well as apply effective sensors. There could be information that cannot be collected through remote sensors, in this case, professional nurses will also be helpful.

\section{Patient-assisting Smart Healthcare System}

The patient-assisting smart healthcare system is becoming more and more popular in recent years [13-18]. Not only could they benefit patients, the caring provided by the systems will also lessen physicians' pressure. The current patient-assisting smart healthcare system is divided into 2 categories. Basic healthcare monitoring system and monitoring for specific diseases.

\section{Basic Healthcare Monitor}

Basic healthcare monitor aims at monitoring basic healthcare information such as heartbeat, heart rate, blood pressure, sleep quality, exercise statistics, and so on. The system also suggests lifestyles based on the data collected, remind exercise and give a warning when the person has a risk to get any disease.

Currently, this kind of systems is still in developing phase. They are helpful in terms of helping people to get better lifestyles. When they are mature, disease prevention will be possible, people's life quality and lifespan will be enhanced tremendously.

\section{Disease-specific Healthcare Monitor}

Disease-specific healthcare monitoring is one of the keys for remote healthcare. They are designed to monitor specific diseases, which are usually high-risk diseases. When the monitored person is dangerous, the monitor will send warning data to both the patients and physicians. In this case, an in-time rescue will be applied, and the chance for the person to survive increases.

As the world population ages, high-risk diseases such as arteriosclerosis, cardiovascular diseases, heart disease, stroke become popular. Since lots of aged people with those diseases live alone, disease-specific healthcare monitoring is crucial.

The most important factor that disease-specific monitoring affects is the timeliness of smart healthcare. In-time rescue is made possible through this technology. In addition, the disease-specific monitoring substitute manual monitoring in some cases thus decreases spending on healthcare.

\section{INTERACTION BETWEEN INFRASTRUCTURES}

Current papers about smart cities mainly discuss each part individually. However, the smart city construction needs to be considered as an integrated system where each part of the system communicates and interact with other parts and maximize the effectiveness. In this part, we'll first introduce a novel thinking provided by the Universal Village. Then, the interaction between smart healthcare and smart safety, data warehouse, smart governance, and smart energy \& environment will be discussed.

\section{A. A Novel Idea Proposed by the Universal Village}

Though technology is developing fast, a single technology can hardly make big difference to the society. The Universal Village finds that systematically coordination between technology itself, and between human and technology, make the whole system work more effectively and efficiently. When each party in a system work coordinately, the system can not only fix its bugs but also exert all its potential. With this idea, we'll discuss how could smart healthcare interact with other aspects and how they generate a better result.

\section{B. Interaction with Smart Safety}

As mentioned above, the data platform is the core of the smart healthcare system. Without the data platform, the whole system could be paralyzed. data safety, hereby, is crucial for the big data platform.

The main request to smart safety is to protect the data platform from losing and being stolen, as well as ensure the system to be functional even if the data platform is damaged. The protection includes hardware level, software level, data backup, and data restoring. More details could be found in [19]. In the other way, the data provided by smart healthcare can show the trend of disease outbreak, which could be applied by smart safety system to better protect people from diseases and other aspects.

\section{Interaction with Data Warehouse}

The data warehouse here refers to the city and country level integrated data warehouse that includes information not only restricted to the smart healthcare aspect. With the local smart healthcare data platform communicating with the big data warehouse, each party will get more useful information.

For the smart healthcare system, the high incidence rate for specific diseases can now be connected to other features provided by the big data warehouse, such as location, environment, living habits of local people, and so on. Thus, better diagnosis and treatment could be given to future patients. In addition, the prediction and prevention of diseases outbreak will also be more accurate with more features pulled from the big data warehouse.

\section{Interaction with Smart Governance}

Smart governance can help establish the unified patients' data platform between hospitals or at least increase the compatibility between each hospitals' platforms. Related laws and regulations should also be given to prevent data abusing.

One of the biggest problems for the current smart healthcare data platforms is that they are independent of each other. Communication between them is hard not only because of technical problem but also because they are unwilling to share data with each other. If the smart governance could be applied and boosts the process of the unification between the platforms, the whole smart system will benefit from it. 


\section{E. Interaction with Smart Energy \& Environment}

Since sensors, data servers, AI assistant will be used in the smart healthcare system, the smart energy, and environment techniques can be used to provide clean and renewable energy, protect garbage metabolized by the system, and find a balance point between the environmental friendliness and the energysupplying. In addition, environmental related data provided by the smart environment platform can be used in the smart healthcare to leverage the accuracy of diagnosing, as well as the disease preventing.

\section{CASE STUdies}

In this part, the smart healthcare construction in Birmingham and Chicago will be discussed.

\section{A. Birmingham}

There are 5 ways that Birmingham applies to make build smart healthcare system [20].

The first method that Birmingham applies is to build personalized digital healthcare system.

The second way is to connect healthcare parties such as family, community, doctors, and hospitals together to maximize the effectiveness of healthcare.

The third approach is to establish digital platform that prioritizes the aged population [21].

The fourth way is to provide challenge-based innovation program for healthcare.

The fifth method is to leverage data to make better healthcare environment including building models, predicting, preventing, providing accessible open data for the citizens, and so on.

\section{B. Chicago}

\section{Chicago Health Atlas}

Chicago Health Atlas focuses on the construction of data platform of smart healthcare [22]. Chicago Health Atlas focuses not only on getting correct data but also on the accessibility and transparency of the data collected. They also help the population to understand the data using techniques such as visualization and comparison over time and communities.

People can not only benefit their health condition from the data directly, but also find opportunities to start a business, and provide services and caring to others. Chicago Health Atlas leverages data to uncover healthcare conditions, diseases propagation, and connect people by giving information.

\section{Healthy Chicago 2.0}

The healthy Chicago 2.0 project considers the Chicago city as a whole and aims at providing a better healthcare system for the local population [23].

The project uses 4 ways to provide better healthcare for the citizens. The first way is to address equity. As an import factor that affects healthcare quality, equity can maximize the benefit of the citizens by giving cares to those who need the healthcare most first. The second way is to connected physicians, government, Non-governmental organizations (NGOs), patients, and other related parties together to provide systematic solutions to healthcare problems. The third aspect is to address better education, economic development, infrastructures construction since the healthcare is influenced by those factors in the long term. The fourth way is to leverage data to provide better diagnosis, treatment, protection, and prevention.

\section{VISION}

The current implementation for the smart healthcare system is far from the proposed version. Even though some cities have a relatively complete healthcare system, the interaction between smart healthcare and other aspects is far from complete. However, the parties in the society know that the smart city system will be more powerful when working integrated as a whole. The integration step will come soon.

In the future, the implementation of the system provided above will be complete, and healthcare will be more affordable, equal to each individual, and technologies will be better leveraged to make the healthcare more accurate and effective.

In 50 years, after the implementation of the current designed system, the lifespan of the population will be extended tremendously. People's psychological health will gain more attention then. As the development of AI, and smart Agency, smart home, and so on, personal robotics will be possible and be applied to the smart healthcare field. They can talk and stay with human beings, aiming at providing better caring both physically and psychologically.

\section{REFERENCES}

[1] World Health Organization Database. http://apps.who.int/nha/database/Select/Indicators/en. [Accessed: 21-Jul2018].

[2] H. Demirkan, "A Smart Healthcare Systems Framework," IT Professional, vol. 15, no. 5, pp. 38-45, 2013.

[3] Institute of Medicine. 2001. Crossing the Quality Chasm: A New Health System for the 21st Century. Washington, DC: The National Academies Press. https://doi.org/10.17226/10027.

[4] "Global Health Observatory (GHO) data: Density of physicians (total number per 1000 population, latest available year)," World Health Organization, 22-Feb-2018. [Online]. Available: http://www.who.int/gho/health_workforce/physicians_density/en/. [Accessed: 21-Jul-2018].

[5] Diana Alexandra. Dumitrescu, "the incumbent crisis of population aging - security implications.," Romanian Review of Political Sciences \& International Relations, vol. 15, no. 1, pp. 55-63, 2018.

[6] J. D. Snell, "Cardiac device with patient-triggered storage of physiological sensor data," 21-May-1996.

[7] M. R. Costanzo and S. Pamboukian, "Device-Based Patient Management," Cardiac Resynchronization Therapy, pp. 404-418.

[8] H. Demirkan, “A Smart Healthcare Systems Framework," IT Professional, vol. 15, no. 5, pp. 38-45, 2013.

[9] A. Amato and A. Coronato, "An IoT-Aware Architecture for Smart Healthcare Coaching Systems,”2017 IEEE 31st International Conference on Advanced Information Networking and Applications (AINA), 2017. 
[10] P. Raj, A. Raman, D. Nagaraj, and S. Duggirala, "Big Data Analytics for Healthcare," Computer Communications and Networks HighPerformance Big-Data Analytics, pp. 391-424, 2015.

[11] W. Raghupathi and V. Raghupathi, "Big data analytics in healthcare: promise and potential," Health Information Science and Systems, vol. 2, no. 1, Jul. 2014.

[12] P. Gupta, D. Agrawal, J. Chhabra, and P. K. Dhir, "IoT based smart healthcare kit," 2016 International Conference on Computational Techniques in Information and Communication Technologies (ICCTICT), 2016.

[13] G. Manogaran, R. Varatharajan, D. Lopez, P. M. Kumar, R. Sundarasekar, and C. Thota, "A new architecture of Internet of Things and big data ecosystem for secured smart healthcare monitoring and alerting system," Future Generation Computer Systems, vol. 82, pp. 375-387, 2018.

[14] P. Rai, P. S. Kumar, S. Oh, H. Kwon, G. N. Mathur, V. K. Varadan, and M. P. Agarwal, "Smart healthcare textile sensor system for unhinderedpervasive health monitoring," Nanosensors, Biosensors, and Info-Tech Sensors and Systems 2012, 2012.

[15] H. J. Baek, G. S. Chung, K. K. Kim, and K. S. Park, "A Smart Health Monitoring Chair for Nonintrusive Measurement of Biological Signals," IEEE Transactions on Information Technology in Biomedicine, vol. 16, no. 1, pp. 150-158, 2012.
[16] S. Manikandan, A. S. Kulkarni, and S. R. S, "IoT based Low Power Wearable ECG Monitoring System," Current Signal Transduction Therapy, vol. 13, 2018.

[17] K. Aziz, S. Tarapiah, S. H. Ismail, and S. Atalla, "Smart real-time healthcare monitoring and tracking system using GSM/GPS technologies," 2016 3rd MEC International Conference on Big Data and Smart City (ICBDSC), 2016.

[18] H. Alemdar and C. Ersoy, "Wireless sensor networks for healthcare: A survey," Computer Networks, vol. 54, no. 15, pp. 2688-2710, 2010.

[19] G. Lafuente, "The big data security challenge," Network Security, vol. 2015, no. 1, pp. 12-14, 2015.

[20] Caprotti, F., Cowley, R., Flynn, A., Joss, S., \& Yu, L. (2016) Smart-Eco Cities in the UK: Trends and City Profiles 2016. Exeter: University of Exeter (SMART-ECO Project).

[21] N. Bryant, N. Spencer, A. King, P. Crooks, J. Deakin, and S. Young, "IoT and smart city services to support independence and wellbeing of older people," 2017 25th International Conference on Software, Telecommunications and Computer Networks (SoftCOM), 2017.

[22] Chicago Health Atlas. [Online].

Available: https://www.chicagohealthatlas.org/about. [Accessed: 24-Jul2018].

[23] Chicago Health Atlas. [Online].

Available: https://www.chicagohealthatlas.org/healthy-chicago. [Accessed: 24-Jul-2018]. 